\title{
Series of high-frequency slowly drifting structures mapping the flare magnetic field reconnection
}

\author{
M. Karlický^ \\ Astronomical Institute of the Academy of Sciences of the Czech Republic, 25165 Ondřejov, Czech Republic \\ Received 30 August 2003 / Accepted 28 November 2003

\begin{abstract}
Rare series of the slowly drifting structures observed during two solar flares (April 11, 2001 and March 18, 2003) in the $0.8-4.5 \mathrm{GHz}$ frequency range are studied. Their time-frequency evolution is compared with topological and X-ray characteristics of the flares. Based on recent modelling of the magnetic field reconnection in the bursting and intermittent regime, it is proposed that these slowly drifting structures map the flare magnetic field reconnection. In such a scenario the drifting structures correspond to the radio emission from primary and secondary plasmoids which are formed in the extended current sheet due to tearing and coalescence processes and they move upwards in the solar atmosphere. An increase and decrease of the frequency drifts of the drifting structures in the initial and decaying flare phases are interpreted as an increase and decrease of the reconnection rate, respectively. On the other hand, individual bursts in the drifting pulsating structures are considered to be radio manifestations of separate electron beams accelerated during the secondary tearing processes. The observed characteristic times are compared with those theoretically predicted and the basic plasma parameters in the flare process are estimated.
\end{abstract}

Key words. plasmas - Sun: flares - Sun: radio radiation - Sun: activity

\section{Introduction}

Slowly drifting radio structures, observed at the beginning of the eruptive solar flares in the $1.5-0.6 \mathrm{GHz}$ frequency range, have been found to be radio signatures of plasmoid ejection (Karlický \& Odstrčil 1994; Kliem et al. 2000; Khan et al. 2002; Karlický et al. 2002). (Most of the slowly drifting structures presented in this paper are so-called drifting pulsating structures (DPS) (Kliem et al. 2000). In the following we refer to these structures mostly as "drifting structures" because it is shorter and more general - see also Karlický et al. 2002.)

Hudson et al. (2001) identified a rapidly moving hard X-ray source, observed by the HXT (Hard X-ray Telescope) of the Yohkoh satellite, associated with the moving microwave source and the plasmoid ejection seen in images observed by the SXT (Soft X-ray Telescope) on board Yohkoh. An association with a high-frequency slowly drifting burst was also reported.

Similarly, Kundu et al. (2001) identified two moving Yohkoh soft X-ray ejecta associated with moving decimetric/metric radio sources observed by the Nançay radioheliograph.

Based on the magnetohydrodynamic (MHD) numerical simulations, Kliem et al. (2000) suggested that each individual burst in the slowly drifting pulsating structure is generated by superthermal electrons, accelerated in the peak of the electric field in the quasi-periodic and bursting regime of the

\footnotetext{
* e-mail: karlicky@asu.cas.cz
}

magnetic field reconnection. Furthermore, the global slow negative frequency drift of the structure was explained by a plasmoid propagation upwards in the solar corona towards lower plasma densities.

Usually only one drifting structure is observed at the beginning of some solar flares in association with a plasmoid ejection. In this paper, we present for the first time the events characterized by a series of slowly drifting structures. These events are first described, then analyzed and finally interpreted using the flare model with the plasmoid ejection.

\section{Observations}

\subsection{April 11, 2001 event}

In April 11, 2001 the GOES satellite observed the soft X-ray event which started at 12:56 UT, with its maximum at 13:26 UT and which ended at 13:49 UT. This event was classified as M2.3 and localized in the active region NOAA AR 9415. According to $\mathrm{H} \alpha$ observations the $1 \mathrm{~F}$ flare was observed at 13:01 UT (start), 13:21 (max), and 14:23 UT (end) in the position S22W27.

As can be seen in Figs. 1-3 and Table 1, a rare series of high-frequency slowly drifting structures was observed during the April 11, 2001 flare in the $0.8-2.0 \mathrm{GHz}$ frequency range by the Ondřejov radiospectrograph (Jiřička et al. 1993). Usually only one such a structure is recorded at the beginning of some eruptive flares. The slowly drifting structure shown in Fig. 2, 

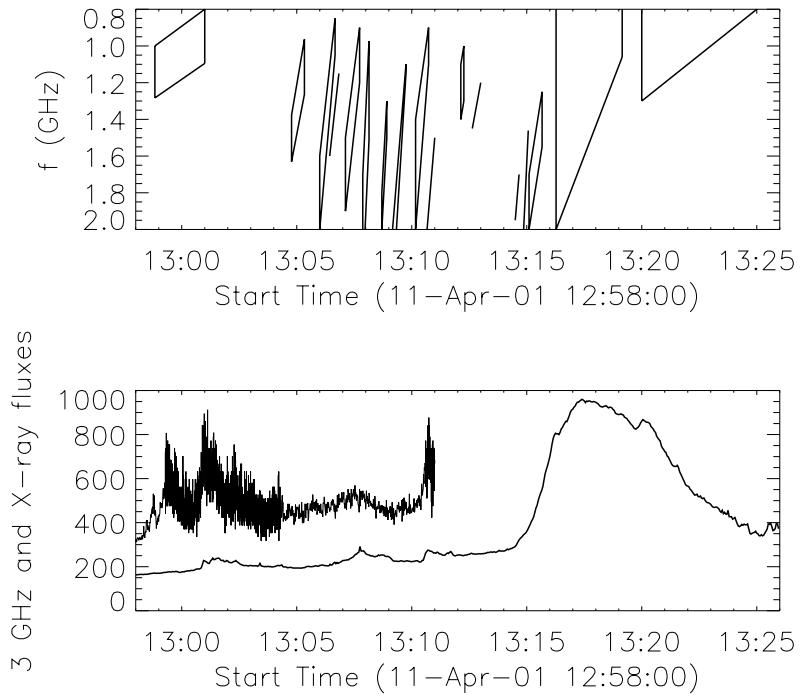

Fig. 1. Time evolution of high-frequency slowly drifting structures (outlines of emission structures in the time-frequency plane) (top), and $3 \mathrm{GHz}$ radio and HXRS (Fárník et al. 2001) 19-29 keV fluxes (in arbitrary units) (bottom) observed during the April 11, 2001 flare. Due to spacecraft night, the HXRS observation ends at 13:11 UT; and after 13:04 UT the time resolution of the observation was changed from $0.2 \mathrm{~s}$ to $1 \mathrm{~s}$.

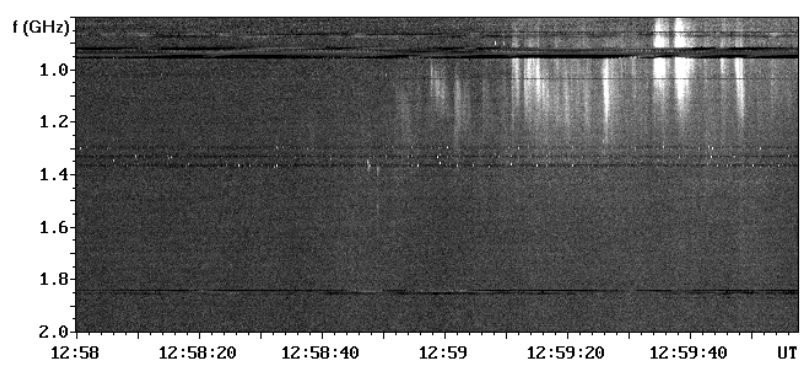

Fig. 2. High-frequency slowly drifting structure observed by the Ondřejov radiospectrograph at the very beginning of the April 11, 2001 flare, at 12:58-13:00 UT.

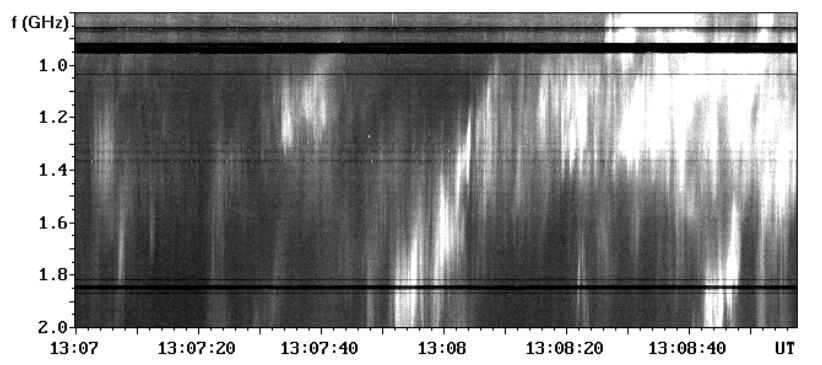

Fig. 3. Example of high-frequency slowly drifting structures observed by the Ondřejov radiospectrograph in April 11, 2001 at 13:07-13:09 UT.

which was recorded at the very beginning of this flare, can be considered as an examle of such typical cases. In a relatively narrow frequency range it consists of many fast drift radio bursts with the characteristic repetition time of seconds. The structure as a whole drifts slowly towards lower frequencies.

But during this event we recorded a series of slowly drifting structures. Their global evolution during the flare is
Table 1. Slowly drifting structures observed in the $0.8-2.0 \mathrm{GHz}$ frequency range during the April 11, 2001 flare. All these drifting structures consist of the fast drift bursts - FDB. L and $\mathrm{H}$ mean the low and high frequency boundaries of the drifting structure (see text).

\begin{tabular}{cccc}
\hline \hline Time & $\begin{array}{c}\text { Freq. range } \\
\text { (UT) }\end{array}$ & $\begin{array}{c}\text { Freq. drift } \\
(\mathrm{GHz})\end{array}$ & Remarks \\
(MHz s & \\
\hline 12:58:50-13:01:00 & $1.00-0.80$ & -1.5 & L, FDB \\
12:58:50-13:01:00 & $1.29-1.09$ & -1.5 & H, FDB \\
13:04:46-13:05:20 & $1.38-0.96$ & -12.3 & L, FDB \\
13:04:46-13:05:20 & $1.63-1.26$ & -10.9 & H, FDB \\
13:06:00-13:06:40 & $1.60-0.85$ & -18.7 & L, FDB \\
13:06:00-13:06:40 & $2.00-1.05$ & -23.7 & H, FDB \\
13:06:26-13:06:50 & $1.60-1.15$ & -18.7 & FDB \\
13:07:07-13:07:44 & $1.50-0.90$ & -16.2 & L, FDB \\
13:07:07-13:07:44 & $1.90-1.20$ & -18.9 & H, FDB \\
13:07:52-13:08:09 & $1.70-0.97$ & -42.9 & L, FDB \\
13:07:58-13:08:09 & $2.00-1.51$ & -44.5 & H, FDB \\
13:08:42-13:08:55 & $1.80-1.30$ & -38.5 & L, FDB \\
13:08:42-13:08:55 & $2.00-1.50$ & -38.5 & H, FDB \\
13:09:10-13:09:45 & $2.00-1.10$ & -25.7 & L, FDB \\
13:09:20-13:09:45 & $2.00-1.15$ & -34.0 & H, FDB \\
13:10:10-13:10:44 & $1.40-0.90$ & -14.7 & L, FDB \\
13:10:10-13:10:44 & $2.00-1.10$ & -26.5 & H, FDB \\
13:10:40-13:11:00 & $2.00-1.50$ & -25.0 & FDB \\
13:12:08-13:12:16 & $1.40-1.30$ & -12.5 & L, FDB \\
13:12:08-13:12:16 & $1.10-1.00$ & -12.5 & H, FDB \\
13:12:38-13:13:00 & $1.45-1.20$ & -11.4 & FDB \\
13:14:30-13:14:40 & $1.95-1.70$ & -25.0 & FDB \\
13:14:52-13:15:04 & $2.00-1.46$ & -45.0 & FDB \\
13:15:06-13:15:40 & $1.70-1.25$ & -13.2 & L, FDB \\
13:15:06-13:15:40 & $2.00-1.55$ & -13.2 & H, FDB \\
13:16:16-13:19:09 & $2.00-1.06$ & -5.4 & H, FDB \\
13:18:00-13:25:00 & $1.30-0.80$ & -1.2 & H, FDB \\
\hline & & &
\end{tabular}

presented schematically in the upper part of Fig. 1. For comparison, the time evolution of the $3 \mathrm{GHz}$ radio and HXRS (Fárník et al. 2001) 19-29 keV X-ray flux are added in the bottom panel of Fig. 1. The flare started at 12:58:50 UT with the drifting structure having an instantaneous bandwidth of $400 \mathrm{MHz}$ and a relatively slow global frequency drift $\left(-1.5 \mathrm{MHz} \mathrm{s}^{-1}\right)$. Then in the time interval of 13:04-13:12 UT a group of drifting structures having higher frequency drifts $\left(-12--45 \mathrm{MHz} \mathrm{s}^{-1}\right)$ followed. The repetition time of these drifting structures was about $45 \mathrm{~s}$. The flare ended in this frequency range by an another group of drifting structures with their frequency drifts changing from $-45 \mathrm{MHz} \mathrm{s}^{-1}$ to $-1.2 \mathrm{MHz} \mathrm{s}^{-1}$ at the end. For more details about variations of frequency drifts of the slowly drifting structures, see Table 1, where the times and frequency drifts of the low (L) and high $(\mathrm{H})$ frequency boundaries of the 


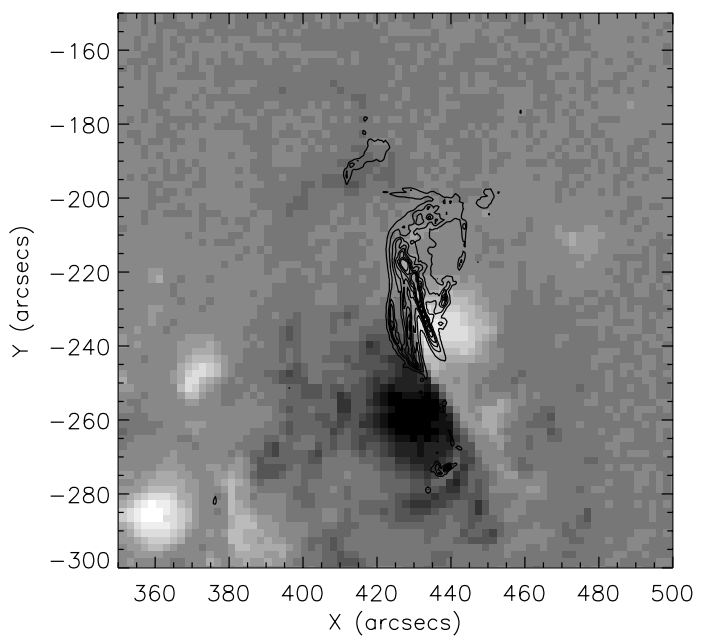

Fig. 4. Trace $171 \AA$ image observed at 13:15:28 UT (contours), April 11, 2001 superposed on the MDI/SOHO line-of-sight magnetic field observed at 12:48:02 UT (grey scale). The white areas correspond to positive magnetic field polarity.

drifting structures are summarized. If the drifting structures had very narrow width, then no designations ( $\mathrm{L}$ or $\mathrm{H})$ are used in this table.

All drifting structures in this flare were composed of many fast drifting quasi-periodic features (bursts) having the characteristic time $\leq 1 \mathrm{~s}$, usually with very high (not measurable) frequency drifts. Their drifts were measurable only in a few cases, as for example in the initial drifting structure (Fig. 2), where pulses with frequency drifts of $-200-+450 \mathrm{MHz} \mathrm{s}^{-1}$ can be recognized.

A very interesting situation can be seen in the radio spectrum (Fig. 3), where several drifting structures are shown. For example one structure (at 13:07:52-13:09:00 UT, with the bandwidth $400 \mathrm{MHz}$ ) drifts towards lower frequencies with the frequency drift of about $-45 \mathrm{MHz} \mathrm{s}^{-1}$ and then its drift stops and further drifting structures drift to and over this stopped structure (e.g. at 13:08:50 UT).

To know the magnetic field topology of the flare, the TRACE (Transition Region And Coronal Explorer) $171 \AA$ image at 13:15:28 UT was superposed on the MDI/SOHO (Michelson Doppler Imager/Solar and Heliospheric Observatory) magnetogram (Fig. 4) and the corresponding extrapolation of the magnetic field in the current-free approximation was made (Fig. 5). As can be seen the flare was located along the magnetic neutral line in the northern part of the NOAA 9415 active region (with magnetic field of sunspots of -1426 and $1220 \mathrm{G}$ ), in the part where the magnetic field reached intensities of -314 and $310 \mathrm{G}$.

\subsection{March 18, 2003 event}

In March 18, 2003 the GOES satellite observed the soft X-ray event which started at 11:51 UT, had its maximum at 12:08 UT and ended at 12:20 UT. This event was classified as X1.5 and localized in the active region NOAA AR 0314. According to $\mathrm{H} \alpha$ observations the 1B flare was observed at 11:56 UT (start), 12:07 (max), and 13:11 UT (end) in the position S15W46.

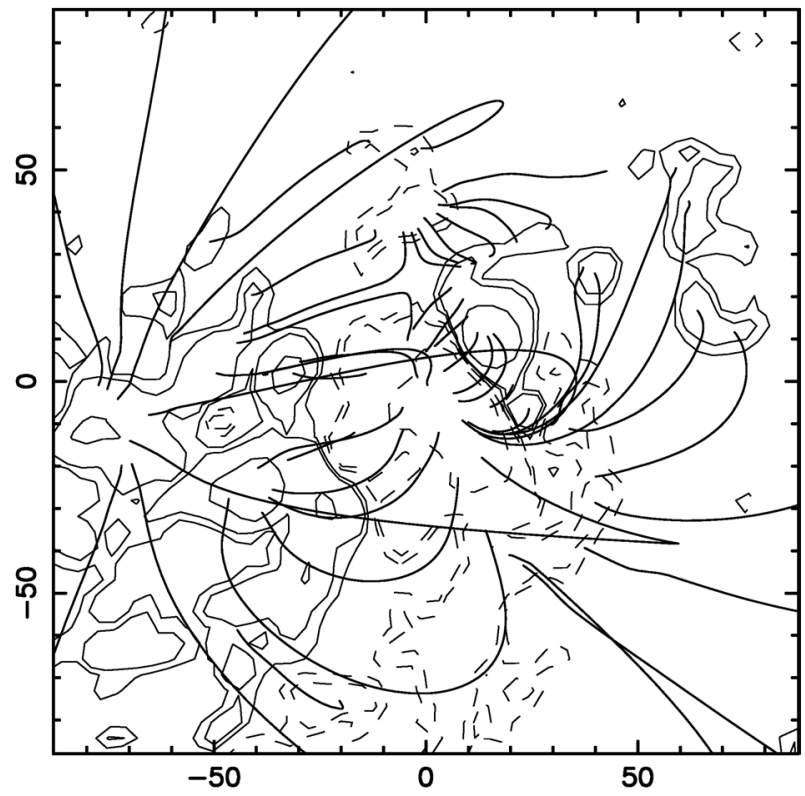

Fig. 5. Current-free extrapolation of magnetic field lines using the $\mathrm{MDI} / \mathrm{SOHO}$ line-of-sight magnetogram observed at 12:48:02 UT, April 11, 2001. Coordinates are in $\mathrm{Mm}$. The magnetic field strength of $\pm 50, \pm 100$, and $\pm 400 \mathrm{G}$ are expressed by full (+) and dashed (-) lines. The centre of this map corresponds to $x=425$, $y=-245$ in the coordinates of Fig. 4.

This flare was also rich in slowly drifting structures. In the $0.8-4.5 \mathrm{GHz}$ range (the range of two Ondřejov radiospectrographs - see Jiřička et al. 1993) the flare started with the initial drifting structure having the frequency drift of $-2.7 \mathrm{MHz} \mathrm{s}^{-1}$ (Fig. 6 and Table 2). Then a group with faster drifting (-4$-21 \mathrm{MHz} \mathrm{s}^{-1}$ ) structures with the characteristic repetition time of $60 \mathrm{~s}$ followed. Among these structures a cloud of narrowband dm-spikes was observed at 12:07:00-12:07:35 UT in the frequency range 1.0-1.7 GHz (Fig. 7). An interesting aspect is that in this time interval a clear double X-ray source (two $\mathrm{X}$-ray sources 20 arcsec apart) in the $12-25 \mathrm{keV}$ energy range was recognized by the RHESSI (Reuven Ramaty High Energy Solar Spectroscopic Imager) satellite; otherwise the RHESSI sources were complex.

After this group of radio drifting structures, and after a gap lasting from 12:10 to 12:15 UT, more slowly drifting structures were observed (their frequency drift is $-0.7-$ $-3.8 \mathrm{MHz} \mathrm{s}^{-1}$ ). Two simultaneous structures can be seen in the time interval of 12:15-12:25 UT. At the end of this interval (at 12:23:40-12:24:10 UT) in high frequencies (1.9-2.5 GHz) a very rare zebra pattern with four zebra lines was recorded (Fig. 8). These zebra lines have sub-structures, they appear to be composed of many narrowband dm-spikes. It is interesting that this zebra pattern also drifted towards lower frequencies with the frequency drift of about $-3.8 \mathrm{MHz} \mathrm{s}^{-1}$.

Finally, at the ending phase of the flare (at 12:38-12:50 UT) the frequency drift of the structures decreased to zero. The bandwidth of the drifting structure (narrowband continuum - NBC in Table 2) at this time is $200 \mathrm{MHz}$ at $1.3 \mathrm{GHz}$ (see Fig. 9), i.e. the bandwidth/mean frequency ratio is 0.15 . 

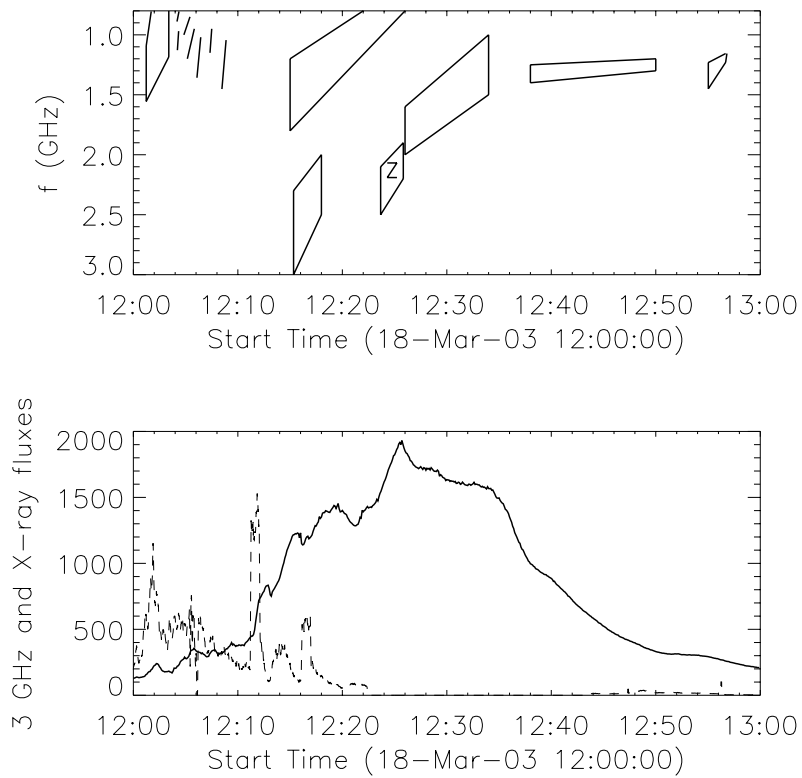

Fig. 6. Time evolution of high-frequency slowly drifting structures (outlines of emission structures in the time-frequency plane) (top), and $3 \mathrm{GHz}$ radio (solid line) and RHESSI 25-50 keV (dashed line) fluxes (in arbitrary units) (bottom) observed during the March 18, 2003 flare. Between 12:22-12:43 UT no RHESSI observations. $\mathrm{Z}$ means the zebra pattern.

For a comparison of the evolution of the drifting structures in this flare, the time profiles of the $3 \mathrm{GHz}$ radio and RHESSI 25-50 keV fluxes are added to Fig. 6. Unfortunately between 12:22-12:43 UT there are no RHESSI observations. Note that the $3 \mathrm{GHz}$ radio flux profile indicates quasiperiodic variations in its ascending part (peak time distances are 210, $110,110,190,170,240,150,210,210 \mathrm{~s}$ starting from the peak at 12:02:20 UT), as known for some types of flares (see e.g. Tajima et al. 1987; Fárník \& Karlický 2002).

Also in this event we were interested to know the flare magnetic field topology and we made a superposition of RHESSI 12-25 keV source image (contours) at 12:01 UT on the MDI/SOHO magnetogram observed at 11:11 UT (Fig. 10). Subsequently, the X-ray source was moving to the south-west; from the position $x=690 \operatorname{arcsec}, y=-160 \operatorname{arcsec}$ at 12:01 UT to the position $x=705 \operatorname{arcsec}, y=-165 \operatorname{arcsec}$ at 12:20 UT (for the coordinates, see Fig. 10). The corresponding magnetic field extrapolation based on the MDI/SOHO magnetogram at 11:11 UT is shown in Fig. 11. In the X-ray source position at 12:01 UT the maximum values of the magnetic field is $-860 \mathrm{G}$ and $540 \mathrm{G}$, in the later phase (12:20 UT) these values are lower $\sim \pm 300 \mathrm{G}$.

\section{Discussion}

In the interpretation of the present events we start from the model proposed by Kliem et al. (2000). We interpret the initial drifting structures in both events as plasma radio emission generated by electron beams which are injected into the plasmoid during a quasi-periodic reconnection process in the current sheet below the plasmoid (Fig. 12). The model enables us to estimate the electron plasma density in the initial plasmoids
Table 2. Slowly drifting structures observed in the $0.8-3.0 \mathrm{GHz}$ frequency range during the March 18, 2008 flare. L and $\mathrm{H}$ mean the low and high frequency boundaries of the drifting structure. Substructures: FDB - fast drift bursts, NBC - narrowband continuum.

\begin{tabular}{|c|c|c|c|}
\hline $\begin{array}{l}\text { Time } \\
\text { (UT) }\end{array}$ & $\begin{array}{l}\text { Freq. range } \\
\quad(\mathrm{GHz})\end{array}$ & $\begin{array}{l}\text { Freq. drift } \\
\left(\mathrm{MHz} \mathrm{s}^{-1}\right)\end{array}$ & Remarks \\
\hline 12:01:14-12:01:43 & $1.90-0.80$ & -10.0 & L, FDB \\
\hline $12: 01: 14-12: 03: 24$ & $1.55-1.20$ & -2.7 & $\mathrm{H}, \mathrm{FDB}$ \\
\hline 12:04:11-12:04:22 & $0.89-0.80$ & -8.2 & FDB \\
\hline 12:04:13-12:04:20 & $1.12-0.97$ & -21.4 & FDB \\
\hline $12: 04: 50-12: 05: 25$ & $1.00-0.85$ & -4.3 & FDB \\
\hline $12: 05: 10-12: 05: 50$ & $1.20-0.95$ & -6.2 & FDB \\
\hline 12:06:05-12:06:27 & $1.36-1.00$ & -16.4 & FDB \\
\hline $12: 07: 20-12: 07: 30$ & $1.15-0.95$ & -20.0 & FDB \\
\hline 12:08:29-12:08:52 & $1.45-1.04$ & -17.8 & FDB \\
\hline $12: 15: 00-12: 22: 00$ & $1.20-0.80$ & -0.9 & L, FDB \\
\hline $12: 15: 00-12: 26: 00$ & $1.80-0.80$ & -1.5 & $\mathrm{H}, \mathrm{FDB}$ \\
\hline $12: 15: 20-12: 18: 00$ & $2.30-2.00$ & -1.9 & L, FDB \\
\hline $12: 15: 20-12: 18: 00$ & $3.00-2.50$ & -3.1 & $\mathrm{H}, \mathrm{FDB}$ \\
\hline $12: 23: 40-12: 25: 50$ & $2.10-1.90$ & -1.5 & L, zebra \\
\hline $12: 23: 40-12: 25: 50$ & $2.50-2.00$ & -3.8 & $\mathrm{H}$, zebra \\
\hline $12: 26: 00-12: 34: 00$ & $1.60-1.00$ & -0.8 & L, FDB \\
\hline $12: 26: 00-12: 34: 00$ & $2.00-1.50$ & -0.7 & $\mathrm{H}, \mathrm{FDB}$ \\
\hline 12:38:00-12:50:00 & $1.25-1.20$ & -0.07 & L, FDB \\
\hline 12:38:00-12:50:00 & $1.40-1.30$ & -0.14 & $\mathrm{H}, \mathrm{NBC}$ \\
\hline $12: 55: 00-12: 56: 40$ & $1.23-1.15$ & -0.8 & $\mathrm{~L}, \mathrm{NBC}$ \\
\hline $12: 55: 00-12: 56: 40$ & $1.45-1.23$ & -2.2 & $\mathrm{H}, \mathrm{NBC}$ \\
\hline
\end{tabular}

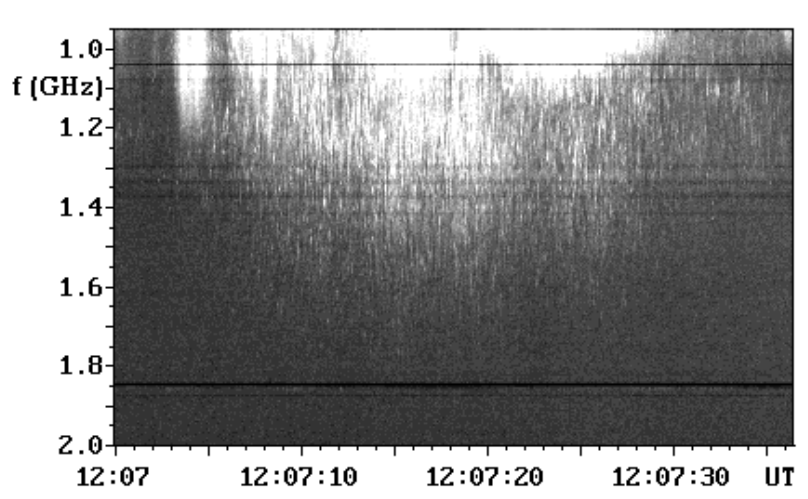

Fig. 7. Narrowband dm-spikes observed at 12:07:00-12:07:35 UT, March 18, 2003 by the Ondřejov radiospectrograph.

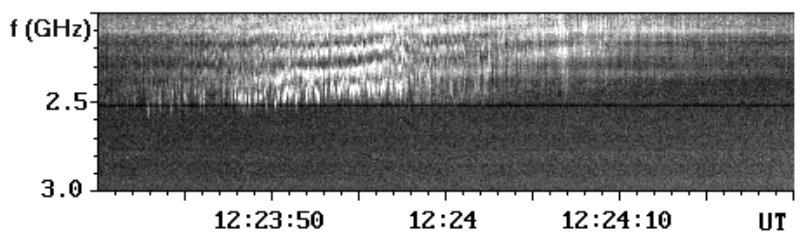

Fig. 8. High-frequency zebra pattern observed at 12:23:3012:24:30 UT, March 18, 2003 by the Ondřejov radiospectrograph. 


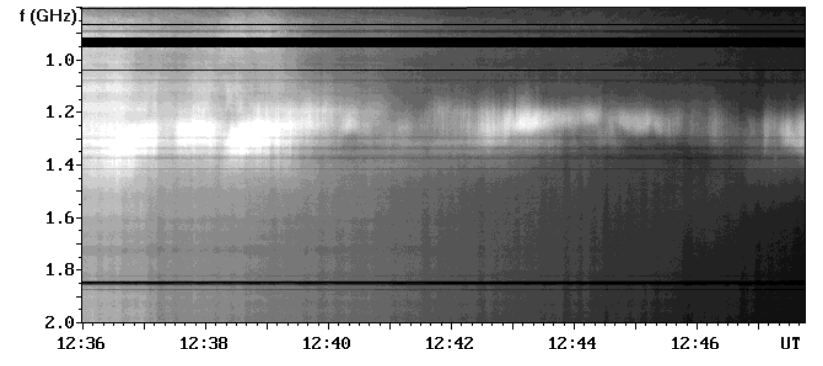

Fig. 9. Narrowband slowly drifting structure observed at the end of the March 18, 2003 flare, at 12:36-12:48 UT by the Ondřejov radiospectrograph.

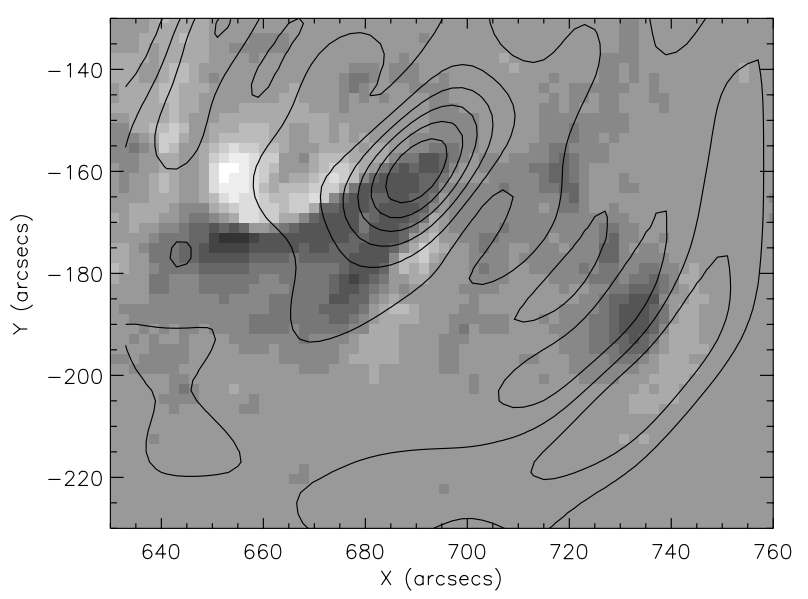

Fig. 10. The RHESSI 12-25 keV source image at 12:01 UT, March 18, 2003 (contours) superposed on the MDI/SOHO line-ofsight magnetic field map observed at 11:11:00 UT (grey scale). The white areas correspond to positive magnetic field polarity.

as, approximately, $n_{\mathrm{e}}=1.77 \times 10^{10} \mathrm{~cm}^{-3}$ (assuming the plasma radio emission at the fundamental electron plasma frequency). According to the model (e.g. Ohyama \& Shibata 1998), the reconnection outflows can reach velocities comparable with the Alfvén speed, thus we expect that these outflows are in a turbulent state. Furthermore, these outflows can be stopped at places below the plasmoid and also at the upper boundaries of underlying closed flare loops, where the termination (fast-mode) shocks may be formed. As suggested by Aurass et al. (2002a) and Bárta \& Karlický (2001) both the termination shocks as well as the turbulent reconnection outflows can be the places of generation of radio emission. While in the case of the radio emission from the termination shocks, the type II-like herring bone structures are expected (Aurass et al. 2002b), the turbulent reconnection outflows can be sources of narrowband dm-spikes (Bárta \& Karlický 2001). Therefore the narrowband dm-spikes observed during the March 18, 2003 event (Fig. 7) can be considered as a further argument supporting the reconnection scenario of the present flares. Moreover, the model of Bárta \& Karlický (2001), by its physical origin, is the same for the narrowband $\mathrm{dm}$-spikes as well as for zebras; their interpretations differ only by the presence of MHD turbulence in the case of spikes. Thus, it is very interesting to see an example of zebra patterns with narrowband dm-spikes as the zebra substructure, which was observed during the March 18, 2003

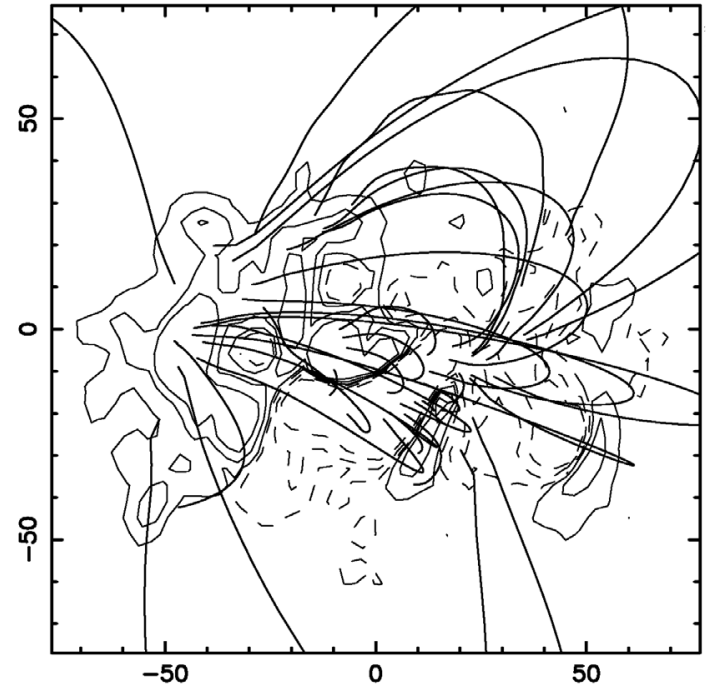

Fig. 11. Current-free extrapolation of magnetic field lines using the MDI/SOHO magnetogram observed at 11:11:00 UT, March 18, 2003. Coordinates are in $\mathrm{Mm}$. The magnetic field strength of $\pm 20, \pm 100$, and $\pm 200 \mathrm{G}$ are expressed by full $(+)$ and dashed ( - ) lines. The centre of this map corresponds to $x=680, y=-155$ in the coordinates of Fig. 10.

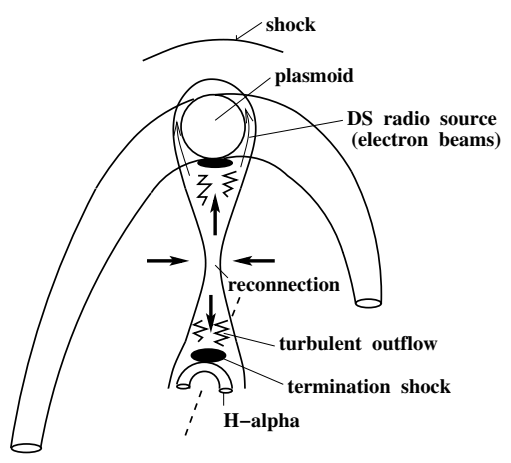

Fig. 12. Flare scenario: initial phase, DS means the drifting structure.

event at 12:23:40-12:24:10 UT (Fig. 8). Such bursts naturally can be expected in the model by Bárta \& Karlický (2001). Furthermore, as shown in the papers by Sawant et al. (2002) and Karlickỳ (2003), if the harmonic number of the zebra pattern lines is determined, the magnetic field in the radio source can be estimated. Assuming in accordance with the mentioned papers that in the present case the zebra line with the highest frequency corresponds to the fifth harmonic of the electron gyro-frequency, then a magnetic field strength of $180 \mathrm{G}$ follows.

The present flares are unique due to an observation of series of the drifting structures. In principle, in the bursting and quasi-periodic regime of the dynamic reconnection in the current sheet, a set of plasmoids can be formed as a result of the tearing and coalescence instabilities (Kliem et al. 2000). These processes even have a cascading form: secondary tearing, tertiary tearing, and so on, always on shorter and shorter spatial scales (Tanuma et al. 2001; Shibata \& Tanuma 2001). Furthermore the plasmoids formed can merge into bigger plasmoids. Tanuma et al. (2001) also showed that an increase of 
the velocity of the plasmoid ejection leads to an increase of the reconnection rate of the reconnection process.

We use these facts for the interpretation of the observed series of slowly drifting structures. We propose that each drifting structure corresponds to some plasmoid (see Fig. 13). Electron beams are accelerated in localized current sheets between neighboring plasmoids and they penetrate into plasmoids, and along their trajectories they generate individual fast drift bursts in specific slowly drifting structures. A series of drifting structures maps the general evolution of the underlying reconnection process. Their negative drifts correspond to motions of the plasmoids along the flare current sheet upwards in the solar atmosphere. This drift varies, expressing the variations of the reconnection rate. After some maximum of the frequency drift (reconnection rate), the frequency drift and thus the reconnection rate decrease at the ending phase of the flares. Using this scenario we can interpret the time behavior of the interacting drifting structures at 13:07:52-13:09:00 UT (April 11, 2001) as a radio manifestation of the coalescence of neighboring secondary plasmoids.

Now, let us consider time scales of the plasmoid formations and electron beam acceleration from the point of view of the dynamic magnetic reconnection in the current sheet (Tanuma et al. 2001) for the April 11, 2001 flare. The characteristic time scale of the drifting structures is $\sim 45 \mathrm{~s}$, the density derived from the drifting structures (radio emission at the fundamental frequency) is $n_{\mathrm{e}}=2.8 \times 10^{10} \mathrm{~cm}^{-3}$. For an assumed magnetic field of the order of $100 \mathrm{G}$, the Alfvén speed is $1300 \mathrm{~km} \mathrm{~s}^{-1}$. The negatively drifting structures indicate that the whole flare magnetic field structure moves upwards. But due to non-stationarity of the flare atmosphere in the low atmospheric layers it is difficult to estimate the velocities from frequency drifts of the slowly drifting structures. For an illustration, assuming the atmosphere in hydrostatic equilibrium with a temperature of $1 \mathrm{MK}$ (the density scale height is $H=50000 \mathrm{~km}$ ), the maximum frequency drift of the drifting structure $-45 \mathrm{MHz} \mathrm{s}^{-1}$ gives a velocity of about $2500 \mathrm{~km} \mathrm{~s}^{-1}$. But using the reduced density scale heights as suggested by Aschwanden \& Benz (1986) $\left(H \sim(2-20) \times 10^{3} \mathrm{~km}\right)$, the plasmoid velocity agrees with those measured during the plasmoid ejection at the solar limb (usually hundreds of $\mathrm{km} \mathrm{s}^{-1}$, e.g. Ohyama \& Shibata 1998). In the April 11, 2001 flare Yohkoh soft X-ray images reveal a loop velocity of about $35 \mathrm{~km} \mathrm{~s}^{-1}$ in projection on the solar disk. Because the loop is seen on the disk it is impossible to recognize whether the loop is a driver of the eruption in the sense of the paper by Ohyama \& Shibata (1998). On the other hand, an upper limit for the plasmoid velocity can be estimated from the type II-like burst observed at 13:12-13:15 UT in the 100-200 $\mathrm{MHz}$ range (Potsdam radio spectrum) as $1100 \mathrm{~km} \mathrm{~s}^{-1}$ using $4 \times$ Newkirk (1967) model of the solar atmosphere. In agreement with the event which is presented in Fig. 7 in the paper by Karlický et al. (2002) we think that both the type II burst and drifting structures belong to the same ejection process, but the type II burst manifests its uppermost part (a shock in the super-Alfvenic regime) and the drifting structures (plasmoids) are located below, moving with sub-Alfvenic speed.

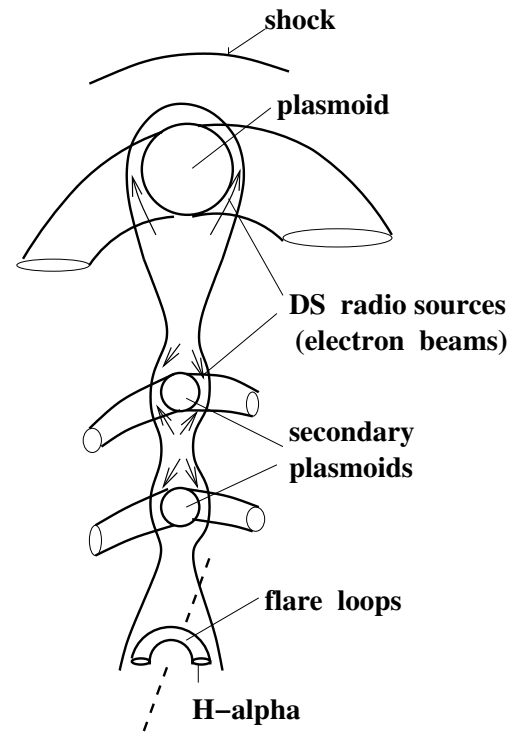

Fig. 13. Flare scenario: main phase, DS means the drifting structure.

We assume that when the first (upper) plasmoid is ejected (Fig. 13) then below this plasmoid a current sheet with an increasing extension in height is formed. The length of this current sheet is $L=v_{\mathrm{p}} \times t$, where $v_{\mathrm{p}}$ is the plasmoid velocity and $t$ is the time. Then at some moment this increasing current sheet becomes unstable due to the tearing instability. The growth rate of this instability is (Priest \& Forbes 2000):

$\gamma=\left(\tau_{\mathrm{d}}^{3} \tau_{\mathrm{A}}^{2} k^{2} l^{2}\right)^{-1 / 5}$

in the range

$\left(\tau_{\mathrm{A}} / \tau_{\mathrm{d}}\right)^{1 / 4}<k l<1$

where $\tau_{\mathrm{d}}=l^{2} / \eta$ and $\tau_{\mathrm{A}}=l / v_{\mathrm{A}}$ are the diffuse and Alfvén transit times, $l$ is the current sheet half width, $\eta$ is magnetic diffusivity, $v_{\mathrm{A}}$ is the Alfvén speed, and $k$ is the wave number of the unstable wave perturbation. Considering now the tearing instability at the wavelength with the maximum growth rate

$\gamma_{\max }=\left(\frac{1}{\tau_{\mathrm{d}} \tau_{\mathrm{A}}}\right)^{1 / 2}$,

then the time at which the lengthening current sheet becomes unstable can be expressed as

$\tau_{\text {onset }}=\frac{2 \pi l\left(\frac{l v_{\mathrm{A}}}{\eta}\right)^{1 / 4}}{v_{\mathrm{p}}}$.

This time for $l=1 \mathrm{~km}, \eta=1 \mathrm{~m}^{2} \mathrm{~s}^{-1}$ corresponding to the temperature $1 \mathrm{MK}$ and two plasmoid velocities $\left(v_{\mathrm{p}}=0.3 v_{\mathrm{A}}\right.$, which is the estimated maximum velocity of the plasmoid (Kliem et al. 2000), and $v_{\mathrm{p}}=0.03 v_{\mathrm{A}}$ ) is shown in Fig. 14 by two dotted lines. The corresponding characteristic times for the tearing instability $\left(\tau_{\text {tear }}=1 / \gamma_{\max }\right)$ and the secondary tearing instability are (Tanuma et al. 2001):

$\tau_{2, \text { tear }}=\left(\frac{l_{2}^{3}}{\eta v_{\mathrm{A}}}\right)^{1 / 2}$, 


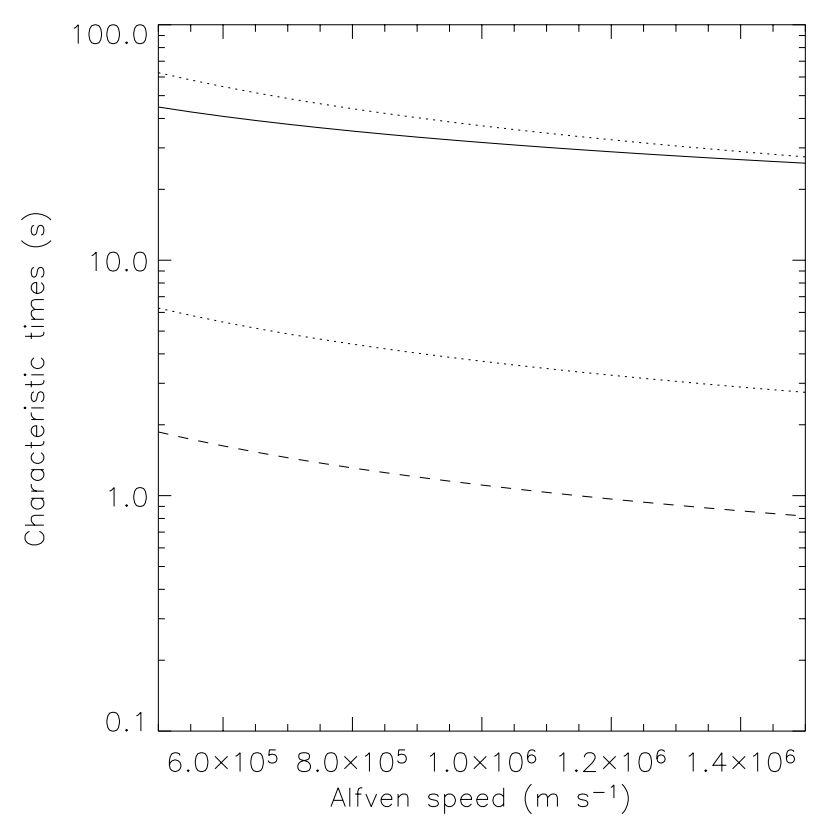

Fig. 14. Characteristic times of the tearing $\tau_{\text {tear }}$ (full line) and secondary tearing $\tau_{2 \text {,tear }}$ (dashed line) instabilities in the flare current sheet having $1 \mathrm{~km}$ thickness vs. the Alfvén speed $v_{\mathrm{A}}$. The times $\tau_{\text {onset }}$ are expressed by dotted lines; the shorter times for the upwards velocity $0.3 v_{\mathrm{A}}$ and longer ones for $0.03 v_{\mathrm{A}}$, respectively.

where

$\left(\frac{l_{2}^{3}}{\eta v_{\mathrm{A}}}\right)^{1 / 2} \leq \frac{L}{v_{\mathrm{A}}}$

and

$L=2 \pi l\left(\frac{l v_{\mathrm{A}}}{\eta}\right)^{1 / 4}$.

The dependence on the Alfvén speed is also plotted in Fig. 14.

In this figure it can be seen that the time of tearing onset $\tau_{\text {onset }}$ (relation 4 ) due to a lengthening of the current sheet is in the 4-40 s interval depending on the upwards velocity of the first plasmoid for the Alfvén speed of $1000 \mathrm{~km} \mathrm{~s}^{-1}$. On the other hand, the tearing time $\tau_{\text {tear }}$ is about $30 \mathrm{~s}$ for the same Alfvén speed. Because the tearing and lengthening processes are not separable ones in our case (e.g. $\tau_{\text {tear }}$ is estimated here for the current sheet with a fixed length), these plots can be used for an interpretation of observations only in a schematic way. For example, we can assume the upwards velocity which gives the time of tearing onset as $15 \mathrm{~s}\left(v_{\mathrm{p}} \sim 0.1 v_{\mathrm{A}}\right)$. Then the tearing lasts about $30 \mathrm{~s}$, which gives the total duration $45 \mathrm{~s}$ $(15+30 \mathrm{~s})$. Simultaneously, the secondary tearing process with a characteristic time of about $1 \mathrm{~s}$ accelerates electron beams which penetrate into plasmoids and appear at radio frequencies as slowly drifting structures (Fig. 13). After these processes the secondary plasmoids can merge with the first (upper) plasmoid or the underlying flare loops. Furthermore, the current sheet continues to grow and once again the tearing instabilities start after some time. If we assume (in a schematic way) a new lengthening of the current sheet and its new tearing we can very roughly estimate a repetition time of these processes as about several tens of seconds, in agreement with the characteristic time of a sequence of the observed drifting structures (45 $\mathrm{s}$ in the April 11, 2001 event). But as mentioned above, the lengthening of the current sheet driven by the first plasmoid and its tearing are not simply separable processes and for a better estimation of this periodicity, numerical simulations are necessary.

Finally, let us comment on a quasiperiodicity which can be seen in the $3 \mathrm{GHz}$ radio flux record in the March 18, 2003 flare (the most frequent time intervals between subsequent $3 \mathrm{GHz}$ peaks are 110 and $210 \mathrm{~s}$ ). These intervals are longer than the repetition time of drifting structures in this case. We think that it might be due to a structure in the reconnection inflow at large scales, or due to multiple triggering of different parts of a complex active region.

\section{Conclusions}

Two examples of a rare series of high-frequency slowly drifting structures were presented. Based on previous studies of these structures and recent models of the magnetic field reconnection it was proposed that these structures map the evolution of the primary and secondary plasmoids which were formed due to the tearing and coalescence instabilities in the current sheet during the reconnection process. This idea can be supported by the presence of further radio bursts which are according to their models connected to the reconnection. Namely, the narrowband dm-spikes and the zebra patterns, having the narrowband dm-spikes as its substructure, can be considered as the radio emissions from the turbulent reconnection plasma outflows. In such a scenario the frequency drift of the drifting structures expresses the motion of plasmoids; the higher frequency drift means a higher velocity of the plasmoid and also a higher reconnection rate of the reconnection process. In both presented flares, variations of the frequency drifts were recognized. After the initial drifting structure with a relatively slow drift rate, in the following times the drift rate reached its absolute maximum, and then at the end of the flare the drift rates of the drifting structures decreased. It was proposed that these variations follow the variations of the reconnection rate. On the other hand, in our scenario, the substructures of the slowly drifting structures (the fast drift bursts with the characteristic time of $1 \mathrm{~s}$ ) map the secondary tearing processes. According to this model, we can even expect burst variations on still shorter and shorter time scales corresponding to the third, forth and higher-order tearing scales as proposed by Tanuma et al. (2001).

Acknowledgements. This paper was finished at the Institute of Space and Astronautical Science at Sagamihara, Japan. The author would like to thank this Institute for inviting him as a guest professor (July-September 2003) and providing him with financial support. $\mathrm{He}$ is grateful to his colleagues for friendly and generous hospitality during his stay in Japan. This research was partly supported also by Grant IAA3003202 of the Academy of Sciences of the Czech Republic. The author thanks to SOHO, TRACE and RHESSI teams for data. The author thanks B. Kliem for helpful comments and suggestions. 


\section{References}

Aschwanden, M. J., \& Benz, A. O. 1986, A\&A, 158, 102

Aurass, H., Karlický, M., Thompson, B. J., \& Vršnak, B. 2002a, in Multi-wavelength observations of coronal structure and dynamics, ed. P. C. H. Martens, \& D. P. Cauffman, COSPAR Coll. Ser., 13, YOHKOH Anniversary Meeting (Pergamon), 401

Aurass, H., Vršnak, B., \& Mann, G. 2002b, A\&A, 384, 273

Bárta, M., \& Karlický, M. 2001, A\&A, 379, 1045

Fárník, F., \& Karlický, M. 2002, in Multi-wavelength observations of coronal structure and dynamics, ed. P. C. H. Martens, \& D. P. Cauffman, COSPAR Coll. Ser., 13, Yohkoh 10th Anniversary Meeting (Pergamon), 169

Fárník, F., Garcia, H., \& Karlický, M. 2001, Sol. Phys., 201, 357

Hudson, H. S., Kosugi, T., Nitta, N., \& Shimojo, M. 2001, ApJ, 561, L211

Jiřička, K., Karlický, M., Kepka, O., \& Tlamicha, A. 1993, Sol. Phys., 147,203
Karlický, M. 2003, Sol. Phys., 212, 389

Karlický, M., \& Odstrčil, D. 1994, Sol. Phys., 155, 171

Karlický, M., Fárník, F., \& Mészarosová, H. 2002, A\&A, 395, 677

Khan, J. I., Vilmer, N., Saint-Hilaire, P., \& Benz, A. O. 2002, A\&A, 388,363

Kliem, B., Karlický, M., \& Benz, A. O. 2000, A\&A, 360, 715

Kundu, M. R., Nindos, A., Vilmer, N., et al. 2001, ApJ, 559, 443

Newkirk, G. 1967, ARA\&A, 5, 213

Priest, E. R., \& Forbes, T. 2000, Magnetic Reconnection (Cambridge, UK: Cambridge University Press), 177

Sawant, H. S., Karlický, M., Fernandes, F. C., \& Cecatto, J. R. 2002, A\&A, 396, 1015

Shibata, K., \& Tanuma, S. 2001, Earth Planets Space, 53, 473

Tajima, T., Sakai, J. I., Nakajima, H., et al. 1987, ApJ, 321, 1031

Tanuma, S., Yokoyama, T., Kudoh, T., \& Shibata, K. 2001, ApJ, 551, 312

Ohyama, M., \& Shibata, K. 1998, ApJ, 499, 934 